\title{
Las reservas de capitalización y nivelación de la nueva Ley del Impuesto de Sociedades y su virtualidad para las entidades sociales
}

\author{
Rosa Fraile FernándeZ \\ Doctora en Derecho \\ Prof. Derecho Financiero y Tributario de la Universidad Rey Juan Carlos
}

DOI: $10.1387 /$ reves. 15259

Fecha de entrada: 20/01/2015

Fecha de aceptación: 10/04/2015

Sumario: I. Planteamiento. II. La nueva Ley del Impuesto sobre Sociedades. III. La reserva de capitalización. IV. La reserva de nivelación. V. Conclusiones. VI. Bibliografía.

\section{Resumen:}

El presente trabajo aborda los nuevos incentivos fiscales al ahorro empresarial a través de la creación de las reservas de capitalización y nivelación incluidas en la recientemente aprobada Ley del Impuesto sobre Sociedades. El tratamiento de la materia se realiza desde la perspectiva de la importancia de la visión de la empresa como un ente cuyo desarrollo se espera perdurable en el tiempo, observando como a las entidades sociales, por ejemplo a las sociedades laborales, este tipo de beneficios fiscales les pueden servir de apoyo a la pretensión de fomentar la capitalización empresarial mediante el incremento de sus fondos propios y con ello contribuir a su saneamiento y competitividad, así como a su viabilidad futura. Objetivos, los citados, íntimamente relacionados con la concepción de la economía social y la previsión futura que se asocia a ellos.

\section{Palabras clave:}

Incentivos fiscales al ahorro, reserva de capitalización, reserva de nivelación, crecimiento sostenible, previsión.

\section{Abstract:}

This article deal with some of the news tax benefits that are include in the lately promulgated Corporate Tax Law in Spain. We are referring to the fiscal saving because of the capitalization of social benefits with the objective of increasing equity. This text addresses the concept of "capitalization reserve" and "levelling reserve", the aim of temporary persistence present in the 
great majority of the social companies and the value of savings in the future stability. Not all entities on the social economy will enjoy this tax benefits, but labour companies constitute an important example of one of the social forms that can take advantage of this legislative newness that look for the business feasibility.

\section{Keywords:}

Tax benefits, sustainable development, increasing equity, new reserves.

\section{Claves ECONLIT}

0410, 0160

\section{Planteamiento}

Diversos son los entes empresariales que operan actualmente en los mercados y diversos son los objetivos que pretenden cumplir a través de ellos quienes los crean y quienes forman parte de su capital. La complejidad de los mercados y la funcionalidad de las empresas suponen una gran riqueza de objetos sociales, tanto expresos como parte de los Estatutos sociales, como de carácter interno de aquellas personas que componen el ente. No toda empresa o negocio tiene un objetivo a largo plazo, pues existen ventajas económicas o de mercado que pretenden ser explotadas al máximo durante un corto periodo de tiempo en el que se espera generarán beneficios y que posteriormente desaparecerán y, con ellas, la viabilidad de la empresa. Del mismo modo, no todo inversor pretende formar parte del capital social de un ente durante un largo periodo, pues, a nadie resultan desconocidas, las operaciones bursátiles intra día.

Sin embargo, nos atrevemos a decir que la mayoría de las empresas, resultan creadas con vocación de permanencia en el tiempo. Lógicamente, una cosa es la permanencia de la empresa y otra la rotación en la titularidad del capital social. Entre estas empresas con clara vocación de permanencia en el tiempo, cabe señalar a aquellas que forman parte de la economía social. Resulta en todo punto lógico que cuando trabajadores y propietarios de una empresa representan al mismo colectivo sea previsible presuponer el deseo de durabilidad, más allá de la manifestación que de ello se haga en el objeto social. Es claro que la liquidación por insolvencia de una empresa resulta traumática para sus trabajadores; obvia- 
mente también lo es para los socios, pero se trata de planos diferentes. Pues bien, ante una cooperativa laboral que cierra, no solo desaparecen los puestos de trabajo, sino también las posibilidades lucrativas de los socios propietarios de la empresa y, como sabemos, en estos escenarios son, con carácter general, las mismas personas quienes pierden su trabajo, y con él los ingresos presentes y futuros, así como sus ahorros, lo que no solo conlleva la pérdida de ingresos futuros sino también la pérdida de aquellos ingresos pasados que fueron invertidos en el proyecto fallido. $\mathrm{La}$ misma consideración merecen las sociedades laborales, menos privilegiadas fiscalmente que las cooperativas, pero con especialidades propias en comparación con el entramado empresarial general.

Es precisamente por lo expuesto que entendemos que las reservas que en este artículo vamos a tratar tienen especial transcendencia para este tipo de negocios en que socios y trabajadores responden, con casi total unicidad a unos propósitos comunes. Los propietarios elegirán un equipo directivo con el que se sientan cómodos y al que dejarán claro que la permanencia en el tiempo es un objetivo prioritario, por encima incluso del cobro de dividendos. Pues el socio trabajador de una cooperativa puede ver sus intereses satisfechos si obtiene el pago que merece por su trabajo, sin buscar, como ocurre en ocasiones en otro tipo de negocios, la obtención de beneficios empresariales a costa de reducir los costes laborales. Asimismo consideramos que acontece con los propietarios del capital de las sociedades laborales, en que su trabajo en la empresa supone que la recompensa por sus acciones y por su tarea vayan íntimamente relacionados.

Este texto va a versar sobre las reservas que, introducidas por la nueva Ley del Impuesto de Sociedades, suponen ventajas fiscales para las empresas previsoras. Obviamente, cada ente empresarial puede destinar a reservas la cuantía que estime oportuna, una vez estén satisfechas las reservas legales. Tras la entrada en vigor de la Ley 27/2014, de 27 de noviembre, del Impuesto sobre Sociedades, el destino de ciertas cantidades a dos tipos de reservas que se definen expresamente en la norma, supone, no solo una previsión en pos del futuro de la empresa y su viabilidad, sino también el ahorro de costes fiscales relacionados con este impuesto. De este modo, el legislador ha querido premiar el ahorro empresarial, ahorro que, como veremos, podrá ser destinado a la superación de momentos de crisis posteriores.

Nos estamos refiriendo a la reserva de capitalización y a la reserva de nivelación, que, si bien, no tienen el mismo ámbito objetivo de aplicación, ni el mismo régimen fiscal, cumplen en ambos casos el citado objetivo de primar fiscalmente a aquellas empresas que empleen políticas de previsión futura. A nuestro juicio, estas políticas, sin duda, facilitarán la permanen- 
cia en el tiempo de tales negocios ${ }^{1}$. Como ya hemos señalado, por el tipo de titulares del capital social, estas reservas resultan de especial interés a las empresas en que socios y trabajadores tienen los mismos intereses, en las cuales consideramos que la permanencia en el tiempo y la viabilidad futura son objetivos prioritarios.

Como es conocido, tanto sociedades laborales como cooperativas tienen sus propios regímenes fiscales especiales, recogidos en sus propias normas específicas. La Ley 4/1997, de 24 de marzo, de Sociedades Laborales, es la que viene regulando estas especificidades en materia de sociedades laborales, incluyendo las cuestiones aplicables tanto a las sociedades anónimas laborales como a las sociedades limitadas laborales. Esta norma exige, en su artículo 14, la creación de una reserva especial. De esta forma se prevé la dotación al Fondo especial de reserva del 10 por ciento de los beneficios de cada ejercicio en que estos se produzcan. Esta reserva, como es sabido, es una reserva adicional. Esto es, las sociedades laborales deberán constituir las reservas que la Ley exige para aquellas sociedades que no tienen la condición de laborales y además, deberán constituir el Fondo especial de reserva, este no supone un porcentaje del capital, que una vez se alcance haya cumplido su objeto, sino que debe dotarse todos y cada uno de los ejercicios en que la empresa obtenga un resultado positivo.

Por su parte, la norma que regula el régimen fiscal de las cooperativas a nivel estatal es la Ley 20/1990, de 19 de diciembre, sobre Régimen Fiscal de las Cooperativas. Como bien es conocido por todos los lectores de esta publicación, estas entidades gozan de un régimen fiscal más beneficioso que el de las sociedades laborales y, en contraposición, deben cumplir con mayor número de requisitos para poder tener la consideración de cooperativas protegidas o especialmente protegidas. Se presenta un verdadero régimen especial en materia de Impuesto de Sociedades, que, por su extrema diferenciación con el régimen general, no se incluye siquiera en el texto del impuesto, sino en la específica norma citada. A las cooperativas se les exige crear dos reservas adicionales. El llamado Fondo de educación y promoción y el Fondo de reserva obligatorio. En Madrid, por ejemplo, conforme a la Ley 4/1999, de 30 de marzo, de cooperativas de la Comunidad de Madrid, el Fondo de Educación y promoción debe

1 Según hemos podido leer en "Tributación de las empresas: baja al 25\% y al 20\% en algunos casos", en Especial Directivos-Coyuntura Empresarial, n. ${ }^{\circ} 1649$, Sección de Noticias, Ed Wolters Kluwer España, 2014: «Se pretende favorecer la creación de reservas propias para contribuir al menor endeudamiento. La conversión de deuda en capital evitará los concursos de acreedores. Es una de las pocas decisiones que sigue los consejos del "comité de expertos" para la reforma fiscal, cuyas recomendaciones tenian mayor alcance.» 
ser del 5 por ciento y la reserva obligatoria del 20 por ciento, en ambos casos haciendo referencia a los resultados cooperativos. En este punto es cada una de las Leyes autonómicas la que fija el porcentaje que deben representar estos fondos, así como qué beneficios se deben tener en cuenta para el cálculo de la cuantía a reservar. Bien tomando en cuenta los beneficios cooperativos, los extracooperativos, los extraordinarios o todos ellos.

Con esto podemos observar cómo, tanto las sociedades laborales como las sociedades cooperativas, ya se ven obligadas por sus normas específicas a dotar una parte considerable de sus beneficios a reservas. Evidentemente, toda reserva tiene la finalidad de preservar una cantidad económica a solventar posibles eventos o problemas futuros. No obstante, como sabemos, nada impide que las cantidades que se destinen a reservas sean mayores que las legalmente exigidas. Las reservas voluntarias se contemplan expresamente en la Ley de Sociedades de Capital así como en las diversas normas de las Comunidades Autónomas relativas a las cooperativas. Así pues, no es una novedad que las empresas destinen mayor parte de sus beneficios a reservas que aquella cuantía que se establece legalmente como obligatoria.

Por ello vamos a analizar estas nuevas reservas previstas por la reciente Ley del Impuesto de Sociedades y cuya dotación permitirá a las empresas que la efectúen obtener determinados beneficios fiscales, en concreto, la reducción de las bases de cálculo de dicho impuesto y, por lo tanto, la reducción de la cuota a pagar en concepto de Impuesto sobre sociedades.

No obstante, queremos indicar antes de empezar a desgranar tales reservas que estas novedades legislativas del impuesto de sociedades no serán aplicables a las sociedades cooperativas, como fundamentaremos más adelante. No podemos explicar con claridad la motivación de este asunto, más allá de que estas entidades ya gozan de un privilegiado entramado de ventajas fiscales. Los incentivos fiscales que se estudiarán en este texto, serán pues aplicables a las sociedades laborales, así como a otro tipo de entidades sociales que tributen conforme al artículo 29.1 LISS, es decir, al tipo de gravamen general o al reducido a causa de ser entidades de nueva creación.

\section{La nueva Ley del Impuesto de Sociedades}

El 28 de noviembre de 2014 fue publicada en el BOE la Ley 27/2014, de 27 de noviembre, del Impuesto sobre Sociedades. Esta norma se incluye dentro de la actual reforma fiscal que podemos resumir 
en la promulgación de la citada Ley, junto con la Ley 26/2014, de 27 de noviembre, por la que se modifican la Ley 35/2006, de 28 de noviembre, del Impuesto sobre la Renta de las Personas Físicas, el texto refundido de la Ley del Impuesto sobre la Renta de no Residentes, aprobado por el Real Decreto Legislativo 5/2004, de 5 de marzo, y otras normas tributarias y la Ley $28 / 2014$, de 27 de noviembre, por la que se modifican la Ley 37/1992, de 28 de diciembre, del Impuesto sobre el Valor Añadido, la Ley 20/1991, de 7 de junio, de modificación de los aspectos fiscales del Régimen Económico Fiscal de Canarias, la Ley 38/1992, de 28 de diciembre, de Impuestos Especiales, y la Ley 16/2013, de 29 de octubre, por la que se establecen determinadas medidas en materia de fiscalidad medioambiental y se adoptan otras medidas tributarias y financieras. Como vemos, se ha producido una reforma de carácter general en relación con los tributos estatales en cuanto a las figuras impositivas a las que afecta. Hemos visto el trabajo del legislador tanto sobre la tributación directa como sobre la indirecta, aunque solo la Ley del Impuesto de Sociedades ha sido reelaborada por completo.

El principal objetivo de la promulgación de la nueva ley del impuesto de sociedades, a través de la Ley 27/2014, ha sido la modernización global del impuesto. La anterior normativa, recogida en el Real Decreto Legislativo 4/2004, de 5 de marzo, por el que se aprueba el texto refundido del Impuesto de Sociedades, fue objeto de numerosas modificaciones desde su entrada en vigor. Todas estas modificaciones, como señala la propia exposición de motivos de la nueva Ley, han sido individualmente consistentes, aunque no han ido acompañadas de una revisión global. Es esta revisión global la que se ha antojado indispensable al legislador en la actualidad, pretendiendo otorgar con ella «un nuevo impulso modernizador y competitivo para el sector empresarial español».

Podemos señalar que el mecanismo del impuesto no ha variado, como tampoco lo hizo con la entrada en vigor del Texto Refundido de 2004 respecto a la legislación previa. Entre los objetivos que presenta la nueva normativa se encuentra el fundamental de favorecer, con carácter primordial, la competitividad empresarial y garantizar el crecimiento sostenido de la actividad económica. A este crecimiento sostenido, sin duda, viene a afectar no solo la reducción del tipo impositivo, cuestión que remarca la exposición de motivos, sino también, según nuestra consideración, esas ventajas fiscales que se otorgarán a quienes creen las reservas que ocuparán el núcleo de nuestra exposición. Como también expresa la motivación de la norma, no se puede olvidar a la pequeńa y mediana empresa, como elemento característico del tejido empresarial español que requiere de la implantación de normas que permitan impulsar su crecimiento y consolidar la competitividad. 
Asimismo, el legislador ha señalado como parte de estos objetivos de la Ley del Impuesto sobre Sociedades, junto con el típico de neutralidad, igualdad y justicia, los de simplificación del impuesto, adaptación a la normativa comunitaria, estabilidad de los recursos y consolidación fiscal, la limitación de deducibilidad de los gastos financieros a fin de controlar el endeudamiento, la seguridad jurídica y la lucha contra el fraude.

Entre las novedades que destaca la exposición de motivos, mencionamos en este punto, aquellas que vincularán el desarrollo de este texto. Citando el texto legal «es objeto de eliminación la deducción por reinversión de beneficios extraordinarios, y la recientemente creada deducción por inversión de beneficios, sustituyéndose ambos incentivos por uno nuevo denominado reserva de capitalización, y que se traduce en la no tributación de aquella parte del beneficio que se destine a la constitución de una reserva indisponible, sin que se establezca requisito de inversión alguno de esta reserva en algún tipo concreto de activo. Con esta medida se pretende potenciar la capitalización empresarial mediante el incremento del patrimonio neto, y, con ello, incentivar el saneamiento de las empresas y su competitividad. Asimismo, esta medida conjuntamente con la limitación de gastos financieros neutraliza en mayor medida el tratamiento que tiene en el Impuesto sobre Sociedades la financiación ajena frente a la financiación propia, objetivo primordial tras la crisis económica y en consonancia con las recomendaciones de los organismos internacionales.» Además, continúa la exposición de motivos, "con destino exclusivo para la pequeña y mediana empresa destaca como novedad la creación de una reserva de nivelación de bases imponibles». Ambas menciones se encuentran ubicadas en el apartado destinado a la modificación efectuada en relación con los incentivos fiscales.

Más adelante, en materia de regímenes especiales, la exposición de motivos vuelve a hacer referencia a la reserva de nivelación. En este punto se indica que la minoración del tipo de gravamen que afecta a estas entidades «se ve acentuada mediante la novedosa reserva de nivelación de bases imponibles negativas, que supone una reducción de la misma hasta un 10 por ciento de su importe. Esta medida resulta más incentivadora que el comúnmente denominado «carry back» en relación con el tratamiento de las bases imponibles negativas, ya que permite minorar la tributación de un determinado período impositivo respecto de las bases imponibles negativas que se vayan a generar en los 5 años siguientes, anticipando, así, en el tiempo la aplicación de las futuras bases imponibles negativas. De no generarse bases imponibles negativas en ese período, se produce un diferimiento durante 5 años de la tributación de la reserva constituida. 
Esta medida pretende favorecer la competitividad y la estabilidad de la empresa española, permitiendo en la práctica reducir su tipo de gravamen hasta el 22,5 por ciento, y, adicionada a la reserva de capitalización anteriormente señalada, incide nuevamente en la equiparación en el tratamiento fiscal de la financiación ajena y propia.»

Realizada esta breve presentación de la nueva Ley del Impuesto de Sociedades en base, principalmente, principalmente al contenido de los apartados I, II y III de la exposición de motivos de la Ley 27/2014, procederemos a desarrollar el comentario acerca de la reserva de capitalización y la de nivelación, cuyo conocimiento consideramos de importancia a fin de poder disfrutar de todas las ventajas fiscales que su constitución puede aportar a las entidades sociales.

\section{La reserva de capitalización}

Como venimos anunciando, una de las novedades introducidas por la Ley 27/2014 es la creación de la reserva de capitalización, que a grandes rasgos, lo que permite a las empresas es destinar el $10 \%$ del total de los beneficios que hayan dedicado al aumento de sus fondos propios, a una reserva indisponible y reducir la base imponible del Impuesto de Sociedades en ese $10 \%$ del aumento de los fondos propios. A diferencia de las desaparecidas deducciones por reinversión de beneficios, no exige una reinversión concreta de estos fondos.

Las cantidades dotadas en esta reserva servirán a la autofinanciación de la empresa y a la reducción de la dependencia de los recursos ajenos, cuestión que se presentaba como uno de los objetivos de la aprobación de la nueva Ley del Impuesto sobre Sociedades. Se trata, pues, de un modo de beneficiar fiscalmente a aquellas compañías que opten por la permanencia de una parte de los beneficios obtenidos en la propia empresa, en lugar de proceder a su distribución entre los socios.

Es el artículo 25 LISS el que se dedica a la definición de esta reserva de capitalización. En primer lugar debemos detenernos a observar el ámbito objetivo de aplicación de esta ventaja fiscal. Primeramente, la norma hace referencia al tipo de gravamen al que deben tributar las empresas para que puedan optar a la reducción de la base imponible que supone la creación de esta reserva. Señala el artículo 25.1 LISS que podrán aplicar esta ventaja «los contribuyentes que tributen al tipo de gravamen previsto en los apartados 1 o 6 del artículo 29 de esta Ley». Esto supone que el ámbito subjetivo de aplicación de este beneficio fiscal solo abarca a los siguientes contribuyentes: Los que tributen conforme al artículo 29.1 LISS que señala que el tipo general de gravamen para los contribuyentes de este Impuesto será el 
25 por ciento. Ahora bien, consideramos que no se debe dejar de lado que la entidad de este primer punto del artículo 29 LISS es mayor que tal inicio. No solo tendrán derecho a aplicar esta ventaja fiscal quienes tributen al tipo general, sino también las entidades de nueva creación que tributen al 15 por ciento, pues es el segundo párrafo del artículo 29.1 LISS el que recoge esta circunstancia. Asimismo, se encontrarán dentro del ámbito objetivo de aplicación de esta ventaja fiscal, las entidades de crédito, así como las entidades que se dediquen a la exploración, investigación y explotación de yacimientos y almacenamientos subterráneos de hidrocarburos, las cuales, conforme al artículo 29.6 LISS tributarán al 30 por ciento.

Si hemos parado a desarrollar esta cuestión es debido a dos motivaciones. La primera, no simplificar el ámbito objetivo de aplicación a quienes tributen al 25 o al 30 por ciento. Pues tal afirmación no es cierta ya que las entidades de nueva creación podrán tributar al 15 por ciento y se engloban en los criterios de aplicación de la reserva y porque no se trata de una materia que realmente tenga que ver con un tipo concreto de tributación, pues si se modificasen los gravámenes presentes en el artículo 29 LISS no dejaría de poder aplicarse la reducción de la base imponible que conlleva la dotación de la reserva. La segunda de las motivaciones es la presentación de la exclusión de las sociedades cooperativas de la aplicación de esta reducción de la base imponible. Esto se debe a que el tipo de gravamen que se aplica a las cooperativas es el recogido en el artículo 29.2 LISS, lo que supone la inoperatividad de la reserva para este tipo de empresas ya que, como hemos expuesto, solo se aplica a quienes tributen conforme a los apartados 1 o 6 del artículo 29 LISS.

La ventaja fiscal para quienes aprovechen este incentivo es la reducción de la base imponible. La norma prevé que esa reducción sea del 10 por ciento del importe de incremento de los fondos propios y exige el cumplimiento de una serie de requisitos. De un lado el incremento de los fondos propios habrá de mantenerse durante un plazo de cinco años desde el cierre del ejercicio en que se aplique la reducción. De otro lado, se debe dotar una reserva por el importe de la reducción, es decir, por el 10 por ciento del incremento de los fondos propios. Esta reserva deberá figurar en el balance de forma separada de cualquier otra y será indisponible también durante el plazo de cinco ańos. Solo queda permitido disponer de esta reserva en tres circunstancias. Cuando el socio se separe de la empresa. Cuando su desaparición se produzca como consecuencia de la aplicación del régimen especial previsto para las fusiones, escisiones, aportaciones de activos, canje de valores y cambio de domicilio social de una Sociedad Europea de un Estado miembro a otro de la Unión Europea.

En cuanto a la cuantía máxima de la reducción de la base imponible, hemos señalado que será del 10 por ciento del aumento de los fondos pro- 
pios. Además se fija legalmente otra limitación. Este 10 por ciento no podría ser superior al 10 por ciento de la base imponible calculada con antelación a la propia reducción que se está tratando y a la compensación de bases imponibles negativas ${ }^{2}$.

Creemos que debe quedar claro que lo que la Ley permite no es la minoración de la base imponible en un $10 \%$, por destinar ese $10 \%$ a una reserva indisponible. Lo que la norma establece es la posibilidad de reducir la base imponible en un $10 \%$ de la cuantía que se haya destinado a aumentar los fondos propios, cuantía que no podrá ser superior al beneficio empresarial. Es decir, solo se podrá minorar la base imponible en un $10 \%$ si todo el beneficio del ejercicio se hubiera destinado al aumento de los fondos propios ${ }^{3}$.

2 Señala el artículo 25.1 LISS en su último párrafo que «en caso de insuficiente base imponible para aplicar la reducción, las cantidades pendientes podrán ser objeto de aplicación en los períodos impositivos que finalicen en los 2 años inmediatos y sucesivos al cierre del período impositivo en que se haya generado el derecho a la reducción, conjuntamente con la reducción que pudiera corresponder, en su caso, por aplicación de lo dispuesto en este artículo en el período impositivo correspondiente, y con el límite previsto en el párrafo anterior».

$3 \mathrm{Si}$ hacemos esta aclaración es porque hemos detectado en la redacción de algunas noticias, que pudiera ser mal entendida esta ventaja fiscal. Así leemos en http://www.bbvacontuempresa.es/a/que-consisten-las-dos-nuevas-reservas-deducibles-del-impuesto-sociedades: «Se crea la reserva de capitalización, para que cualquier empresa pueda destinar a una provisión exenta de impuestos limitada al $10 \%$ de sus beneficios, sin que sea necesario que éstos se reinviertan en activos de la propia empresa», apreciación que se expone sin contener más aclaraciones. En http://www.pymesyautonomos.com/fiscalidad-y-contabilidad/la-reserva-de-capitalizacion-una-medida-adecuada-pero-que-se-queda-corta, se indica: «con este nuevo instrumento las empresas podrán destinar hasta el $10 \%$ de su beneficio a elevar los recursos propios de la sociedad, dinero por el cual no tendrán que tributar.» En http://www.expansion.com/2014/09/01/ pymes/1409585835.html, se puede leer: «esta figura otorga a las pymes la posibilidad de disfrutar de una reducción de otro $10 \%$ de la base imponible por el incremento de fondos propios.» En "Comentario a la Ley 27/2014, de 27 de noviembre, del Impuesto sobre Sociedades», en Boletín Fiscal, n. ${ }^{\circ}$ 12, diciembre de 2014, Editorial Wolters Kluwer Espańa «la nueva reserva de capitalización permite reducir la base imponible en un 10 por 100 por incremento de fondos propios (art. 25).» Por su parte, otras webs de divulgación contienen la expresión: esta reserva supone una reducción de la base imponible del impuesto del 10 por ciento del importe del incremento de sus fondos propios", como aparece, por ejemplo en: http://www.supercontable.com/pag/documentos/comentarios/ nueva_reserva_capitalizacion.html. Esta definición resulta correcta y ajustada a la realidad normativa. Si bien, y aunque resulte un poco más largo, con el fin de no llevar a equívocos al lector, nos posicionamos por dar importancia a una versión que, aunque simplificada, resulte más completa, por ejemplo: «esta reserva se concreta en una reducción en la base imponible del 10\% del importe del incremento de los fondos propios de la entidad, siempre que (i) dicho incremento se mantenga durante un plazo de 5 años y (ii) se dote una reserva por el importe de la reducción, que será indisponible durante dicho plazo de 5 años», obtenida de: Junquera Temprano, A.; "La reforma del Impuesto sobre Sociedades", en Estrategia Financiera, N.o 321, Sección A efectos Legales, Noviembre 2014, Editorial Wolters Kluwer Espańa. 
Nos parece preciso señalar que la norma establece que a efectos de determinar el incremento de los fondos propios no se tendrán en cuenta las aportaciones de los socios, las ampliaciones de capital o fondos propios por compensación de créditos, las ampliaciones de fondos propios por operaciones con acciones propias o de reestructuración, las reservas de carácter legal o estatutario, las reservas indisponibles que se doten por aplicación de lo dispuesto en el artículo 105 de la LISS y en el artículo 27 de la Ley de modificación del Régimen Económico Fiscal de Canarias, los fondos propios que correspondan a una emisión de instrumentos financieros compuestos, ni los fondos propios que se correspondan con variaciones en activos por impuesto diferido derivadas de una disminución o aumento del tipo de gravamen del impuesto.

En vista de este listado de acciones que suponen un aumento de los fondos propios y que, sin embargo, no dan derecho a la aplicación de la reducción de la base imponible, podemos considerar que la reducción de la base imponible se podrá aplicar cuando se aumenten los fondos propios debido a la capitalización de los beneficios sociales, existiendo el derecho a reducir la base imponible en un 10 por ciento de esta capitalización de beneficios, siempre que, además, se dote una reserva indisponible por ese 10 por ciento.

Para finalizar el comentario sobre la nueva reserva de capitalización debemos incluir los efectos del incumplimiento de los requisitos legales. Según dispone el artículo 25.4 LISS, y como suele acontecer en estos casos de pérdida del derecho tras su ejercicio, se habrán de regularizar las cantidades indebidamente deducidas, debiendo ingresarse, junto con la cuota del período impositivo en que tenga lugar el incumplimiento de los requisitos, la cantidad deducida así como los intereses de demora devengados.

\section{La reserva de nivelación}

La reserva de nivelación se configura como la otra de las ventajas fiscales que, como venimos señalando, resultan de aplicación a aquellas empresas que deseen actuar de forma previsora. Reservando una parte de sus beneficios de forma que puedan afrontar en mejor situación posibles escenarios de desaceleración o incluso pérdidas, se obtendrán no solo las ventajas propias de esta actuación, sino también beneficios fiscales. Esta reserva, a diferencia de la anterior, solo resulta aplicable a empresas de reducida dimensión. En este sentido, señala la exposición de motivos que de entre las novedades de la Ley 27/2014 se destaca la eliminación de la escala de tributación tradicional aplicable a las empresas de reducida dimensión, si bien, debido a la reducción de tipos impositivos, que con carácter gene- 
ral opera en la Ley, la entrada en vigor de la nueva LISS supone la minoración del tipo de gravamen que afecta a las pymes. Cierto es el contenido del preámbulo en este punto, las empresas de reducida dimensión ven reducido su tipo de gravamen; no obstante, pierden la diferenciación impositiva en cuanto al tipo respecto a las empresas de régimen general. Se sitúan en situación preferible frente a la normativa anterior, aunque comparativamente han perdido algunos privilegios respecto de aquellas empresas que tributan al régimen general ${ }^{4}$.

Esta reducción del tipo de gravamen que, como hemos señalado, es general y no solo para las pymes, se ve acentuada, en este caso solo para las empresas de reducida dimensión, mediante la creación de la reserva de nivelación. Esta, y de forma exclusiva para empresas consideradas pymes, supone una reducción de la base imponible del Impuesto sobre Sociedades hasta un 10 por ciento. Con esta medida, como veremos, lo que se permite es minorar la tributación del periodo impositivo en curso y afectar esta minoración a las posibles bases imponibles negativas de los años cinco años posteriores. En el satisfactorio supuesto de que no se produzcan tales bases imponibles negativas en los años posteriores al ejercicio del derecho a la reducción de la base imponible en virtud de la dotación a la reserva de nivelación, lo que se va a conseguir por parte de quienes hayan aplicado esta ventaja fiscal es el diferimiento de la tributación de ese 10 por ciento de la base imponible durante cinco ańos.

Según indica el preámbulo de la Ley, y en cuya apreciación estamos totalmente de acuerdo, "esta medida pretende favorecer la competitividad y la estabilidad de la empresa española, permitiendo en la práctica reducir su tipo de gravamen hasta el 22,5 por ciento, y, adicionada a la reserva de capitalización anteriormente señalada, incide nuevamente en la equiparación en el tratamiento fiscal de la financiación ajena y propia.»

Comenzando ya con la exposición de este incentivo fiscal, vamos a tratar en primer lugar, al igual que en el apartado previo, el ámbito subjetivo de aplicación de esta novedosa reserva. Inicialmente podríamos pensar que la reserva de nivelación sí podría ser aplicable a las sociedades cooperativas,

${ }^{4}$ A este respecto, podemos leer en «¿Cómo pagar menos impuestos tras la reforma del impuesto de sociedades?» en Revista Contable, sección Se habla de ..., 8 de octubre de 2014, Ed Wolters Kluwer Espańa, 2014, que "con la rebaja del tipo impositivo general no existe en términos absolutos pérdida de beneficio fiscal por parte de las pymes y que con la introducción de la reserva de nivelación de bases imponibles prevista exclusivamente para pymes se permite en la práctica reducir el tipo de gravamen de estas empresas al 22,5\%, también lo es que las pymes pierden la ventaja comparativa que tienen en la actualidad en relación al tipo de gravamen reducido, ventaja que constituia un beneficio fiscal definitivo a diferencia de la reserva de nivelación que supone únicamente un diferimiento de 5 años para aquellas sociedades que no acaben generando bases imponibles negativas.» 
pues el primer requisito que se enuncia para poder ser beneficiarios de la reducción de la base imponible es ser una entidad de reducida dimensión, característica que cumplen muchas de las sociedades cooperativas. Si bien, el artículo que regula esta reserva, el 105 LISS, indica expresamente que podrán minorar la base imponible conforme se dispone en el precepto, solo aquellas entidades que apliquen en el periodo impositivo el gravamen previsto en el primer párrafo del apartado 1 del artículo 29 LISS. Esta reserva tiene, por tanto, un ámbito subjetivo de aplicación mucho más restringido que la reserva de capitalización. Es requisito esencial que la empresa que pretenda disfrutar del beneficio fiscal sea una entidad de reducida dimensión conforme al artículo 101 LISS y, además, que aplique el tipo de gravamen previsto "en el primer párrafo del apartado 1 del artículo 29». No solo se excluyen, en comparación con la reserva de capitalización, a las empresas cuyo tipo de gravamen se recoge en el artículo 29.6 LISS, sino que aquí también quedan excluidas las que aplican el tipo reducido del 15 por ciento, por ser entidades de nueva creación 5 .

Una vez aclarado qué empresas pueden acogerse a esta novedosa reserva de nivelación, es de importancia resaltar cuál es la ventaja fiscal que ello supone, aunque tal ventaja ya ha sido enunciada en diversas ocasiones a lo largo de este texto. Las empresas podrán minorar su base imponible, en caso de que esta sea positiva, hasta un máximo del 10 por ciento de su importe, con el límite de 1 millón de euros. Esto es, si la empresa obtiene una base imponible de más de 10 millones de euros, la reducción máxima que podrá aplicar será el millón, aunque tal cuantía sea inferior al 10 por ciento. Ahora bien, hemos de reconocer que son pocas las pymes que alcanzan bases imponibles positivas de tal envergadura.

El contribuyente debe dotar una reserva, la reserva de nivelación de bases imponibles, por el importe de la reducción de la base imponible que haya efectuado en el ejercicio. Esta reserva ha de dotarse con cargo a los resultados positivos del ejercicio en que se realice la citada minoración de la base imponible.

La misión principal de esta reserva es la compensación de pérdidas posteriores, concretamente de los cinco años siguientes al ejercicio en que se dote. Por ello, si la entidad tiene bases imponibles negativas, deberá ir cancelando esta reserva en la cuantía necesaria para que la base imponible resulte cero. Si, transcurridos cinco años desde la dotación de la reserva, la

5 El tipo aplicable a las entidades de nueva creación se encuentra regulado en el artículo 29.1 LISS, si bien, no se encuentra incluido en su primer párrafo. Por ello, entendemos aplicable a las empresas de nueva creación que tributen al 15\%, la ventaja fiscal que supone la reserva de capitalización, pero, por virtud de lo dispuesto en la Ley, no podemos considerar aplicable a estos entes la reserva de nivelación. 
empresa no la ha cancelado totalmente por aplicarla a resultados negativos, (bien por haber tenido siempre bases imponibles positivas, bien por haber sido las bases negativas de menor cuantía que la reserva dotada), el importe que permanezca en tal reserva se sumará a la base imponible de ese ejercicio quinto posterior a la dotación.

Esta reserva solo podrá disponerse, con carácter general en dos supuestos. El primero, el de nivelación de bases imponibles negativas de los cinco años posteriores a la dotación de la reserva. El segundo, el de adicción a la base imponible una vez hayan transcurridos los cinco años que la Ley exige. A estos efectos, al igual que hemos anunciado en relación con la reserva de capitalización, no se entenderá que se ha dispuesto de la reserva cuando el socio ejerza su derecho de separación, cuando la reserva se elimine total o parcialmente a causa de una fusión, escisión, aportación de activos, canje de valores o cambio de domicilio social de una Sociedad Europea de un Estado miembro a otro de la Unión Europea.

La norma establece en artículo 105.5 LISS la incompatibilidad de esta reserva con otras. Como podía entenderse sin necesidad de mención expresa, las cantidades que se destinen a la dotación de la reserva de nivelación no pueden ser aplicadas de forma simultánea a la dotación de la reserva de capitalización previamente estudiada, ni tampoco a la reserva para inversiones en Canarias que prevé el artículo 27 de la Ley de modificación del Régimen Económico y Fiscal de Canarias.

Por último, en el apartado sexto del mismo precepto de la Ley del Impuesto sobre Sociedades se establecen los efectos que suponen el incumplimiento de los requisitos que dan derecho a la reducción de la base imponible por esta causa. En este caso, queda previsto que se haya de incluir en la cuota íntegra del periodo impositivo en que tenga lugar el incumplimiento la cuota correspondiente a las cantidades que hayan sido objeto de minoración, esta cuota habrá de incrementarse además, en un cinco por ciento y añadirle los intereses de demora. Los efectos del incumplimiento, como vemos, difieren de lo previsto para la reserva de capitalización, no solo en ese cinco por ciento citado, sino también en que el ajuste no se ha de producir en la base, sino en la cuota. Se distancia así este proceder del habitual, que es el que queda regulado en el artículo 125.3 LISS.

\section{Conclusiones}

Siempre son bienvenidas las modificaciones legislativas que favorecen la viabilidad de las empresas. El ahorro, como acontece en todas las esferas de la economía, supone un balón de oxígeno para el futuro, permitiendo un mayor gasto en un tiempo posterior o una compensación 
de las pérdidas venideras. Es por ello que aplaudimos la inclusión en la nueva Ley del Impuesto sobre Sociedades de ventajas fiscales para las empresas que decidan destinar parte de sus beneficios al aumento de sus fondos propios. Lo que, sin duda, supone dotar de mayor estabilidad al entramado empresarial.

Asimismo, como hemos comentado en el primer apartado de este trabajo, entendemos que este tipo de ventajas son de especial importancia para las empresas sociales en las que, como hemos incidido, existe una vocación de durabilidad. Especialmente, si cabe, destacamos este objetivo de permanencia en el tiempo en las sociedades laborales así como en las cooperativas de trabajadores. No obstante, estas últimas, junto con el resto de cooperativas protegidas no pueden aplicar en pos de obtener ventajas fiscales las reservas aquí estudiadas. De un lado, se ha de reconocer que tales entidades se encuentran sometidas a un régimen fiscal especialmente beneficioso en comparación con el resto, lo cual permite entender los motivos por los que no se ven incluidas en el ámbito subjetivo de aplicación de estos incentivos. No obstante, la propia exposición de motivos de la Ley 27/2014 indica que «es objeto de eliminación la deducción por reinversión de beneficios extraordinarios, y la recientemente creada deducción por inversión de beneficios, sustituyéndose ambos incentivos por uno nuevo denominado reserva de capitalización». Podemos decir, sin miedo a equivocarnos, que en este punto el articulado no es coherente con la exposición de motivos, al menos en relación con las empresas cooperativas. Si nos atrevemos a apuntar esto es debido a que la deducción por reinversión de beneficios extraordinarios ha venido aplicándose a las sociedades cooperativas $^{6}$. Por ello, si esta deducción se sustituye por la reserva de capitalización, esta debería poder aplicarse también a tales entidades.

Más allá de esta cuestión sobre la no aplicación de la deducción a las cooperativas, consideramos interesante, para otras entidades sociales, la aprehensión del funcionamiento de estos incentivos fiscales, que permitirán reducir la cuota del impuesto de sociedades a la vez que aseguran el mantenimiento de cierto ahorro en el patrimonio empresarial.

${ }^{6}$ Debido a las especialidades de la tributación de las cooperativas, la deducción que estaba prevista en el artículo 42 del TRLIS, se debía calcular atendiendo a tales especialidades. Así, por ejemplo, la consulta vinculante a la DGT, V0029-2009, de 12 de enero, indicaba que esta deducción debía realizarse en el caso de las cooperativas agrarias aplicando «el coeficiente que corresponda, a las rentas positivas obtenidas integradas en la base imponible. De ello se deduce que no se tienen en cuenta las rentas positivas obtenidas que no se hayan integrado en la base imponible, como es el caso del 50\% de la parte de los resultados extracooperativos destinados obligatoriamente al Fondo de Reserva Obligatorio, que no se habrán integrado en la base imponible.» 
Dos son estas nuevas reservas que permiten la reducción de la base imponible. La primera de ellas es la reserva de capitalización. Esta ventaja fiscal se configura de forma que aquellas empresas que incrementen sus fondos propios con cargo al resultado del ejercicio podrán ver reducida su base imponible en un 10 por ciento de ese incremento de los fondos propios. Se exige la creación de una reserva, la reserva de capitalización, en la que se ha de incluir ese 10 por ciento de la base imponible que se ha reducido y que deberá permanecer inalterado, salvo determinados supuestos muy concretos, hasta que hayan transcurrido cinco años desde su dotación.

Por su parte, la reserva de nivelación de bases imponibles constituye una ventaja fiscal aplicable exclusivamente a empresas de reducida dimensión. Este incentivo fiscal consiste en que las empresas podrán crear una reserva, la reserva de nivelación, en la que se incluya el 10 por ciento de su base imponible. Este 10 por ciento se reducirá de tal base imponible a efectos del cálculo del impuesto. En caso de que se presenten bases imponibles negativas en los cinco ejercicios siguientes, esta reserva se habrá de ir cancelando hasta dejar la base imponible en cero. En caso de que no existan resultados negativos, la reserva de nivelación se cancelará a los cinco años, debiendo incluirse la cuantía que represente en la base imponible de tal ejercicio. En estos supuestos, la reserva supondrá el diferimiento del impuesto.

Ambos mecanismos, parezcan o no suficientes en pos del objetivo pretendido, suponen incentivos a la viabilidad de las empresas en un futuro, a la previsión en aras de la perdurabilidad en el tiempo, así como a la racionalización del recurso a la financiación ajena ${ }^{7}$. Todo ello a costa de reducir los beneficios inmediatos a repartir entre la titularidad de la empresa. Consideramos que, en tal sentido, han de valorarse positivamente y esperar que, una vez se encuentre verdaderamente implantada la Ley 27/2014, de 27 de noviembre, del Impuesto sobre Sociedades, estos incentivos fiscales sirvan a la estabilidad, y no solo a un ahorro puntual en la cuota del impuesto, de aquellas entidades que opten por aplicarlos.

7 Ya se pueden leer algunos textos doctrinales en que se critica el reducido potencial de la reserva de capitalización, al afectar solo a un pequeño porcentaje de la base imponible, por ejemplo en RODRÍGUEZ RALEA, F.J.; "Deducciones para incentivar la realización de determinadas actividades" (nota previa al capítulo), en VVAA, Fiscalidad Práctica 2014: IRPF, Patrimonio y Sociedades, Ed. Lex Nova, Valladolid, 2014. El mismo comentario es frecuentemente repetido en las diversas webs informativas acerca de la reforma fiscal. 


\section{Bibliografía}

Junquera Temprano, A.; "La reforma del Impuesto sobre Sociedades», en Estrategia Financiera, n. ${ }^{\circ}$ 321, Sección A efectos Legales, Noviembre 2014, Editorial Wolters Kluwer España

Rodríguez Ralea, F.J.; «Deducciones para incentivar la realización de determinadas actividades» (nota previa al capítulo), en VVAA, Fiscalidad Práctica 2014: IRPF, Patrimonio y Sociedades, Ed. Lex Nova, Valladolid, 2014.

"Comentario a la Ley 27/2014, de 27 de noviembre, del Impuesto sobre Sociedades", en Boletín Fiscal, N. ${ }^{\circ}$ 12, Diciembre 2014, Editorial Wolters Kluwer España

"¿Cómo pagar menos impuestos tras la reforma del impuesto de sociedades?" en Revista Contable, sección Se habla de ..., 8 de octubre de 2014, Ed Wolters Kluwer España

"Tributación de las empresas: baja al 25\% y al 20\% en algunos casos», en Especial Directivos-Coyuntura Empresarial, n. ${ }^{\circ}$ 1649, Sección de Noticias, Ed Wolters Kluwer España, 2014.

http://www.bbvacontuempresa.es/a/que-consisten-las-dos-nuevas-reservasdeducibles-del-impuesto-sociedades.html

http://www.expansion.com/2014/09/01/pymes/1409585835.html

http://www.pymesyautonomos.com/fiscalidad-y-contabilidad/la-reserva-decapitalizacion-una-medida-adecuada-pero-que-se-queda-corta.html

http://www.supercontable.com/pag/documentos/comentarios/nueva_reserva_ capitalizacion.html 